\title{
Rigorous and Phenomenological Equations of State
}

\author{
Werner Däppen and Dan Mao \\ Department of Physics and Astronomy, University of Southern California \\ Los Angeles, CA 90089-1342, USA \\ email: dappen@usc.edu \&dmao@usc.edu
}

\begin{abstract}
For solar and stellar modeling, a high-quality equation of state is crucial. But the inverse is also true: the astrophysical data (helioseismic today, asteroseismic tomorrow) put constraints on the physical formalisms, making the Sun and the stars laboratories for plasma physics. One of the main astrophysical benefits from a good equation of state is an improved abundance determination. Recent theoretical progress in the equation of state has involved both rigorous and phenomenological approaches, giving the user a considerable choice.
\end{abstract}

Keywords. Equation of state, Plasmas, Atomic processes, Stars: evolution, Sun: helioseismology

\section{Introduction}

A simple ideal-gas model of the plasma of the solar interior was adequate before helioseismology, but helioseismic and asteroseismic equation-of-state analyses require more sophisticated physical models. In the case of the Sun, the need to go beyond the ideal-gas approximation for helioseismic applications had been recognized in the early 1980s (see Berthomieu et al. 1980; Ulrich 1982; Noels et al. 1984). With the better data that became available towards the end of the 1980s, a clearer picture began to emerge. ChristensenDalsgaard et al. $(1988,1996)$ demonstrated that it was essential to include the Coulomb correction. The relative Coulomb pressure correction peaks in the outer part of the convection zone (about -8 per cent) and in the solar core (about -1 per cent). For solar conditions, the Debye-Hückel (DH) theory is a good approximation for the leading term of the Coulomb correction.

Helioseismic equation-of-state studies typically use solar models based on sophisticated new equations of state, in particular, the ones underlying the two ongoing major opacity re-computation efforts. One of these efforts is the international Opacity Project (OP; see the books by Seaton 1995; Berrington 1997); it contains the so-called Mihalas-HummerDäppen equation of state (Hummer \& Mihalas 1988; Mihalas et al. 1988; Däppen et al. 1988; Nayfonov et al. 1999; Trampedach et al. 2006); hereinafter MHD) and it deals with heuristic concepts about the modification of atoms and ions in a plasma. The other effort is being pursued at Lawrence Livermore National Laboratory by the OPAL group (Iglesias and Rogers 1996; Rogers et al. 1996; Rogers \& Nayfonov 2002); its equation of state is based on a detailed systematic method to include density effects in a plasma.

Although approximate asymptotic techniques (see Christensen-Dalsgaard et al. 1985; Gough 1993) exist to invert solar oscillation frequencies for the internal sound speed, for an accurate analysis of the observations, a fully-fledged, non-asymptotic numerical treatment of the oscillations is mandatory (see Gough et al. 1996). Figure 1 is a typical result of such a numerical inversion (Basu \& Christensen-Dalsgaard 1997). It shows the relative difference (in the sense sun - model) between the squared sound speed obtained from inversion of oscillation data and that of a two standard solar models. The two solar models 


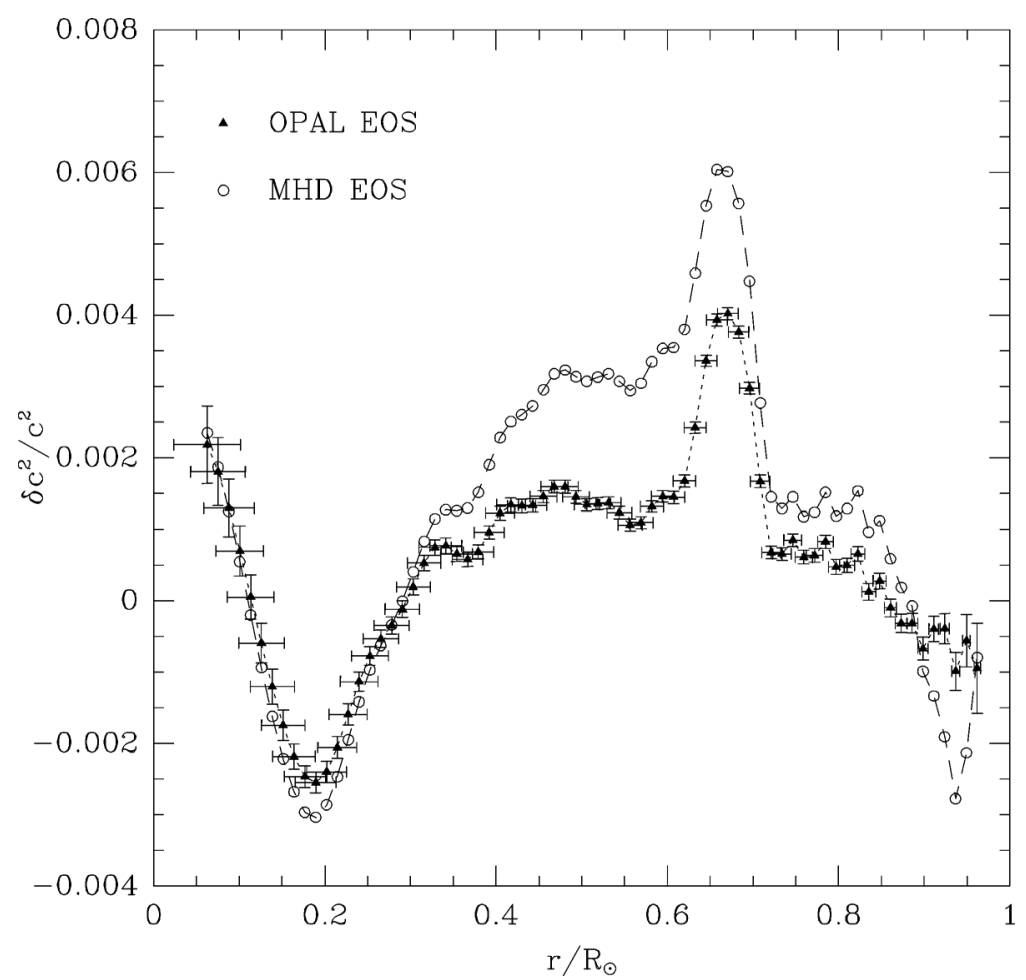

Figure 1. Difference between squared sound speed from inversion and two solar models, which only differ in their equation of state (see text). Figure by S. Basu.

used are identical in all respects except for their equation of state, MHD (circles) and OPAL (triangles), respectively. For the present purpose, we can consider inversion results such as Figure 1 as the data of helioseismology, disregarding the procedure through which they were actually obtained from solar oscillation frequencies. It follows from Figure 1 that in most parts of the sun the OPAL equation of state is a better approximation to reality than MHD, but OPAL needs to be improved as well.

\section{Various equations of state}

With the exception of neutron stars, in stellar interiors the thermal de Broglie wavelength of nuclei, atoms, ions and molecules is always tiny and for them a classical treatment is sufficient. Only electrons have to be treated according to quantum mechanics. At this point there is a bifurcation into two distinct classes of approach, the "chemical picture" and the "physical picture" (see, e.g., the book by Kraeft et al. 1986). While in the more conventional chemical picture bound configurations (atoms, ions and molecules) are introduced and treated as new and independent species, only fundamental particles (electrons and nuclei) appear in the physical picture. In the chemical picture, reactions between the various species occur, and thus the thermodynamical equilibrium must be sought among the stoichiometrically allowed set of concentration variables by means of a maximum entropy (or minimum free-energy) principle. In contrast, the physical picture has the esthetic advantage that there is no need for a minimax principle; the question of bound states is dealt with implicitly through the Hamiltonian describing the interaction between the fundamental particles. 
As mentioned in the Introduction, in most parts of the Sun, OPAL fares better than MHD. However, all OPAL results are so far only available in the form of pre-computed tables, and they have been made for certain chemical compositions of astrophysical relevance (Rogers et al. 1996). In 2005, the most recent OPAL equation of state tables were released. These tables allow low stellar mass down to $0.1 \mathrm{M}_{\odot}$ (Rogers \& Nayfonov 2002). However, it is important to note that the OPAL computer code is proprietary and belongs exclusively to the Livermore group. Therefore, the community cannot use OPAL without recourse to tables. If the goal is to have more flexibility than is possible with mere tables, one solution consist in retrofitting chemical-picture formalisms such that they agree with OPAL. Indeed, Liang (2004) and Liang \& Däppen (2003, 2004) have successfully emulated the OPAL equation of state. A recent extension of that work (Mao 2008) allowed the OPAL emulation for a mixture of hydrogen and helium (see Section 4).

Alternatively, progress can be made by developing more rigorous equations of state. One example is based on the path-integral formalism in the framework of the so-called Feynman-Kac (FK) representation. The FK formalism leads to a virial expansion of the thermodynamic functions in power of the total density of a Coulomb plasma (Alastuey \& Perez 1992; Alastuey et al. 1994, 1995). Like OPAL, this approach was developed in the physical picture. Specific to the Feynman-Kac representation is the equivalence between a point-charge quantum system and a classical one made of extended filaments. The explicit calculation of the thermodynamic function has been carried out up to order $\rho^{5 / 2}$ in total density for general plasmas (Alastuey \& Perez 1996). The finite truncation limits its domain of validity to a temperature and density regime close to the conditions of full ionization (see next section). It is planned to achieve the implemention of FK in solar and stellar models (see Section 5).

\section{Activity versus virial expansions}

In the previous section, we have mentioned that virial expansions limit their domain of validity to a temperature and density regime close to the conditions of full ionization, a restriction to which activity are not subject. We illustrate this different behavior using a simple but typical example. The essential technique can be understood by considering the exact equation of the ideal (non-relativistic) Fermi gas. It is an implicit equation of state, obtained from the grand-canonical partition function (see, e.g. )

$$
\frac{p}{k T}=\frac{1}{\lambda^{3}} f_{5 / 2}(\tilde{z}), \quad \frac{N}{V}=\frac{1}{\lambda^{3}} f_{3 / 2}(\tilde{z})
$$

Here, $p, T, N, V, k$ are pressure, temperature, particle number, volume, and the Boltzmann constant, respectively. The thermal de Broglie length $\lambda$, and the fugacity $\tilde{z}$ are defined based on the Fermi integrals $f_{5 / 2}$ and $f_{3 / 2}$

$$
\lambda=\frac{h}{\sqrt{2 \pi m k T}} ; \tilde{z}=e^{\frac{\mu}{k T}}, f_{5 / 2}(\tilde{z})=\frac{4}{\sqrt{\pi}} \int_{0}^{\infty} d x x^{2} \ln \left(1+\tilde{z} e^{-x^{2}}\right), f_{3 / 2}(\tilde{z})=\tilde{z} \frac{d}{d \tilde{z}} f_{5 / 2}(\tilde{z}) .
$$

Expanding the Fermi integrals as a series in the high-temperature limit, and eliminating the fugacity $\tilde{z}$, one obtains the density (virial) expansion

$$
\frac{p V}{N k T}=1+\frac{1}{2^{5 / 2}} \frac{N \lambda^{3}}{V}+\ldots
$$




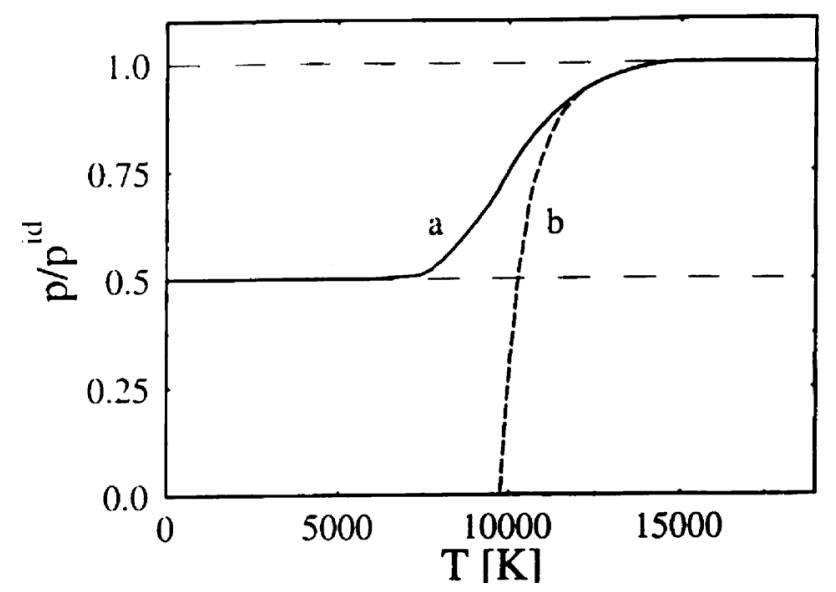

Figure 2. Pressure in a simple reacting system of neutral hydrogen $\mathrm{H}$, recombining to hydrogen molecules $\mathrm{H}_{2}$. $P_{\text {id }}$ refers to fully dissociated hydrogen. The activity expansion (a) correctly describes recombination with just one non-ideal term (equations 3.4,3.5). Without further high-order terms, the corresponding virial expansion (b) (i.e.the reacting analog to the single-species equation 3.3) exhibits catastrophic non-physical behavior (negative pressure!) (Ebeling et al. 1976).

This is a nice and important result, since it gives not only the familiar classical limit of a rigorous quantum result, but it also states the precise physical conditions when that limit is attained. Note that this equation is a convenient density expansion. However, I now show that not eliminating the fugacity also has its rewards. One can write

$$
\begin{aligned}
& \frac{p}{k T}=\frac{1}{\lambda^{3}}\left[\tilde{z}-\frac{1}{2^{5 / 2}} \tilde{z}^{2}+\ldots\right]=z-\frac{\lambda^{3}}{2^{5 / 2}} z^{2}+\ldots, \\
& \frac{N}{V}=\frac{1}{\lambda^{3}}\left[\tilde{z}-\frac{1}{2^{3 / 2}} \tilde{z}^{2}+\ldots\right]=z-\frac{\lambda^{3}}{2^{3 / 2}} z^{2}+\ldots,
\end{aligned}
$$

with the definition of the activity $z$

$$
z=\frac{1}{\lambda^{3}} \tilde{z} .
$$

Note that while these expressions are still for the ideal Fermi gas, from the structure of the formal grand-canonical partition function it is obvious that any real gas has an activity expansion (no need to evaluate the grand-canonical partition function, merely to look at it)

$$
\begin{aligned}
& \frac{p}{k T}=z-b_{2} z^{2}+\ldots, \\
& \frac{N}{V}=z-2 b_{2} z^{2}+\ldots,
\end{aligned}
$$

It is the task of a theory for a specific system to deliver the expansion coefficients. Nevertheless, already before doing that one can convince oneself of an important advantage of the activity formalism when applied to reacting gases (the system of neutral hydrogen $\mathrm{H}$ recombining to hydrogen molecules $\mathrm{H}_{2}$ is the simplest case; a system of protons and electrons recombining to atomic hydrogen is another relatively simple case) (Ebeling et al. 1976). Figure 2 demonstrates that already the lowest order of an activity expansion describes the low-temperature recombination correctly. Although as infinite 
old MHD —
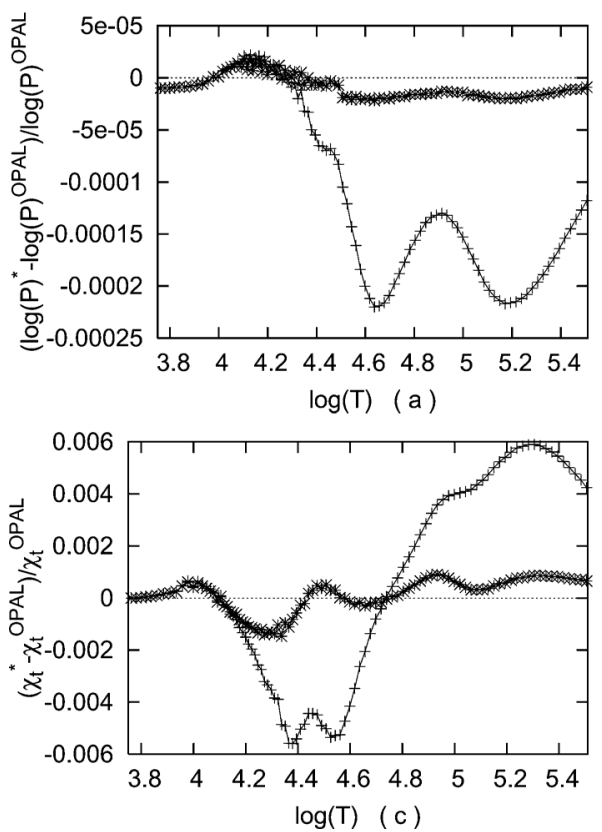

new MHD $\cdots * \cdots$
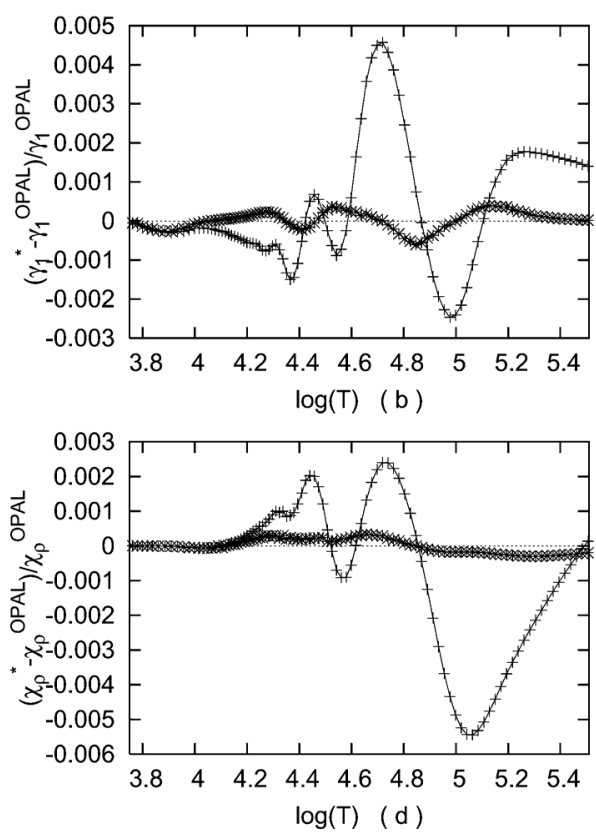

Figure 3. OPAL emulator: relative difference of thermal quantities (a) $\log p$, (b) $\Gamma_{1}$, (c) $\chi_{T}$, and (d) $\chi_{\rho}$ in a solar model, in the sense old MHD - OPAL and new MHD - OPAL, respectively. OPAL is thus represented by the zero line. The quality of the emulation is reflected by the closeness of the new MHD values (large asterisks) to zero.

series, activity and density expansions are strictly equivalent, a density expansion would have to go to very high order merely to describe recombination (or ionization).

The chemical picture can do without the introduction of the activity variable; it is therefore more intuitive. However, the chemical picture is plagued with fundamental weaknesses, such as the heuristic but never rigorous assumption of the existence of atoms in a plasma (for an introduction to current issues see, e.g., Däppen 2004, 2006). Activity expansions (such as OPAL) are not limited by these weaknesses. The OPAL equation of state has demonstrated that activity expansions combine the conceptual advantage of the physical picture with an accurate practical realization, needing to involve only a relatively small number of expansion terms.

\section{Progress with the phenomenological approach: the OPAL emulator}

As part of her PhD thesis, Mao (2008) has upgraded the OPAL emulator mentioned in Section 2, by extending it from pure hydrogen to a hydrogen-helium mixture. As before, the MHD equation of state has been appropriately modified, by adding Coulombscattering terms and a modified internal partition function (the so-called "Planck-Larkin partition function" PLPF, see e.g. Ebeling et al. 1976). The resulting emulator gives excellent agreement with OPAL, as shown in Fig. 3 (here, "old" MHD denotes the usual MHD, "new" MHD the OPAL emulator). It is obvious that the remaining differences between the modified MHD equation of state have become much smaller than those between the original MHD equation of state and OPAL. Work is in progress to release a user-friendly version of the emulator to the community. 


\section{The rigorous FK equation of state}

Although the MHD and OPAL equations of state can be very successful in describing the structure of the Sun and stars, there is still room in improving its precision. Among all the attempts to improve the equation of state, the formalism of path integrals in the framework of the Feynman-Kac (FK) representation promises the most rigorous results, because it contains all non-ideal contributions to the equation of state, systematically, exactly and analytically.

As we have seen in Section 3, the fact that FK is a density (virial) expansion causes a severe limitation of its domain of validity. The plasma has to be nearly fully ionized in order for the method to be valid. Despite this limitation, the FK approach can still be applied to large parts of stellar interiors (see Perez et al. 2008). In the locations where FK can be used, it is especially suited to study effects of screening, bound states, the location of the transition to full ionization, and diffraction and exchange effects. Incidentally, follow-up papers of Perez et al. (2008) will be dedicated to the extension of the FK calculations to higher orders, including the order $\rho^{3}$ in density. This will allow us to take into account exactly the three-body effects occurring in a fairly ionized plasma (for example, in the case of the Sun, the recombination of $\mathrm{He}^{++}$).

\section{Conclusions}

While currently available equations of state give reasonable accuracy for solar and stellar modelers, the observational data have the potential to aim higher, not only for better models, but especially for a maximum use of the Sun and stars to serve as plasma-physics experiments. These experiments are complementary to on-going efforts in terrestrial laboratories.

In the equation of state work, there has been recent progress both on the phenomenological and rigorous side. On the phenomenological side, the result is a useful OPAL emulator. It is based on new terms put in MHD. These terms were directly borrowed from OPAL and have no natural foundation in the chemical picture; therefore they were missing in the original MHD equation of state. (It is unclear if they could ever be found consistently within the MHD approach.) Physically, the upgrade of MHD reflects the fact that MHD, as any typical chemical-picture formalism, describes ionized electrons in the plane-wave approximation. The main added ingredient is based on Coulomb wave functions for the free (scattering) states and using the Planck-Larkin partition function. We have demonstrated that such an upgrade of MHD does indeed bring it very close to OPAL. The resulting OPAL emulator will be a useful tool for solar and stellar modelers, who will be able to use the OPAL equation of state directly, without recourse to the OPAL tables distributed by the Lawrence Livermore National Laboratory. (Currently there is no alternative to the official OPAL equation of state tables, since their source code is not publicly available.)

On the rigorous side, there has been recent progress with an equation of state based on the Feynman-Kac path-integral formalism. Although it is expressed as a density expansion, which limits its application (Section 3), the FK formalism can still be applied to most regions of the solar interior. The localizing power of helioseismology will allow us to test the FK equation of state in the regions of the solar interior where it works well. And since FK is so far the most exact and systematic equation-of-state approach, it will provide a new way to study the influence of various fine physical effects. 


\section{Acknowledgements}

We thank Asher Perez, Forrest Rogers and Hugh DeWitt for stimulating discussions. This work was supported by the grant AST-0708568 of the National Science Foundation.

\section{References}

Alastuey, A. \& Perez, A. 1992, Europhys. Lett. 20, 19

Alastuey, A., Cornu, F., \& Perez, A. 1994, Phys. Rev. E, 49, 1077

Alastuey, A., Cornu, F., \& Perez, A. 1995, Phys. Rev. E, 51, 1725

Alastuey, A. \& Perez, A. 1996, Phys. Rev. E, 53, 5714

Basu, S. \& Christensen-Dalsgaard, J. 1997, Astron. Astrophys., 322, L5

Berrington, K. A. 1997, The Opacity Project, vol. II, Institute of Physics Publishing. Bristol

Berthomieu, G., Cooper, A. J., Gough, D. O., Osaki, Y., Provost, J., \& Rocca, A. 1980, in Hill,

H. A., Dziembowski, W., eds., Lecture Notes in Physics 125, Springer, Heidelberg, p. 307

Christensen-Dalsgaard, J., Gough, D. O., \& Toomre, J. 1985, Science, 229, 923

Christensen-Dalsgaard, J., Däppen, W. \& Lebreton, L. 1988, Nature, 336, 634

Christensen-Dalsgaard, J., Däppen, W., and the GONG Team 1996, Science, 272, 1286

Däppen W., 2004, in Danesy D., ed., Proc. SOHO 14/GONG 2004: Helio- and Asteroseismology:

Towards a Golden Future. ESA SP-559, Noordwijk, p. 261

Däppen W., 2006, J. Phys. A: Math. Gen., 39, 4441

Däppen, W., Mihalas, D., Hummer, D. G. \& Mihalas, B. W. 1988, Astrophys. J., 332, 261

Ebeling W., Kraeft W. D., \& Kremp D., 1976, Theory of Bound States and Ionization Equilibrium in Plasmas and Solids. Akademie-Verlag, DDR-Berlin

Gough, D. O., Kosovichev, A. G., Toomre, J., and the GONG Team 1996, Science, 272, 1296

Gough, D. O. 1993, in Zahn, J.-P., Zinn-Justin, J., eds., Astrophysical Fluid Dynamics, NorthHolland, Amsterdam, p. 399

Huang K., 1963, Statistical Mechanics. John Wiley, New York, Chapt. 14

Hummer, D. G. \& Mihalas, D. 1988, Astrophys. J., 331, 794

Iglesias, C. A. and Rogers, F. J. 1996, Astrophys. J., 464, 943

Kraeft W. D., Kremp D., Ebeling W., \& Röpke G. 1986 Quantum Statistics of Charged Particle Systems, (New York: Plenum)

Liang, A., 2004, "Emulating the OPAL equation of state in the chemical picture formalism", in Equation-of-State and Phase-Transition Issues in Models of Ordinary Astrophysical Matter, edited by V. Celebonovic, W. Däppen, \& D. Gough, AIP Conference Proceedings 731, Melville, New York, 2004, p. 106

Liang, A. \& Däppen, W. 2003, "Modifications of the Equation of State to Achieve Desired Changes in Thermodynamic Quantities," in Proc. SOHO 12/GONG+ 2002 Workshop (ESA SP-517, Noordwijk, The Netherlands), p. 333-336.

Liang, A. \& Däppen, W. 2004, "Emulating the OPAL equation of state", in "Helio- and Asteroseismology: Towards a Golden Future", SOHO14-GONG2004 Meeting held July 12-16 2004 at Yale University, New Haven, CT, USA (ESA Publications Division: Noordwijk, The Netherlands), p. 548.

Mao, D. 2008, PhD Thesis, USC, Los Angeles

Mihalas, D., Däppen, W., \& Hummer, D. G. 1988, Astrophys. J., 331, 815

Nayfonov, A., Däppen, W., Hummer, D. G., \& Mihalas, D. M. 1999, Astrophys. J., 526, 451-464.

Noels, A., Scuflaire, R., \& Gabriel, M. 1984, Astron. Astrophys., 130, 389

Perez, A., Mussack, K., Däppen, W., \& Mao, D. 2008, Astron. Astrophys., submitted.

Rogers F. J. \& Nayfonov A., 2002, Astrophys. J., 576, 1064

Rogers, F. J., Swenson, F. J., \& Iglesias, C. A. 1996, Astrophys. J., 456, 902

Seaton, M. J. 1995, The Opacity Project Vol. I, Institute of Physics Publishing. Bristol

Trampedach R., Däppen W., \& Baturin V. A., 2006, Astrophys. J., 646, 560

Ulrich, R. K. 1982, Astrophys. J., 258, 404 


\section{Discussion}

Christensen-DalsgandD: What is the potential for determining the abundances of $\mathrm{O}, \mathrm{C}, \mathrm{N}$ etc. from their effect on the equation of state in the convection zone, using helioseismology.

DÄPPEN: The size of the modulation of $r_{1}$ by the heavy elements is (marginally) within the power of present-day helioseismology. A preliminary study by C.-H. Lin (2004) has looked at this effect and it was found that $\mathrm{Z}$ weight indeed below the Anders-Grevesse value. 